\title{
Harnwegsinfektion: Phytopharmakon wirkt nachgewiesen antientzündlich
}

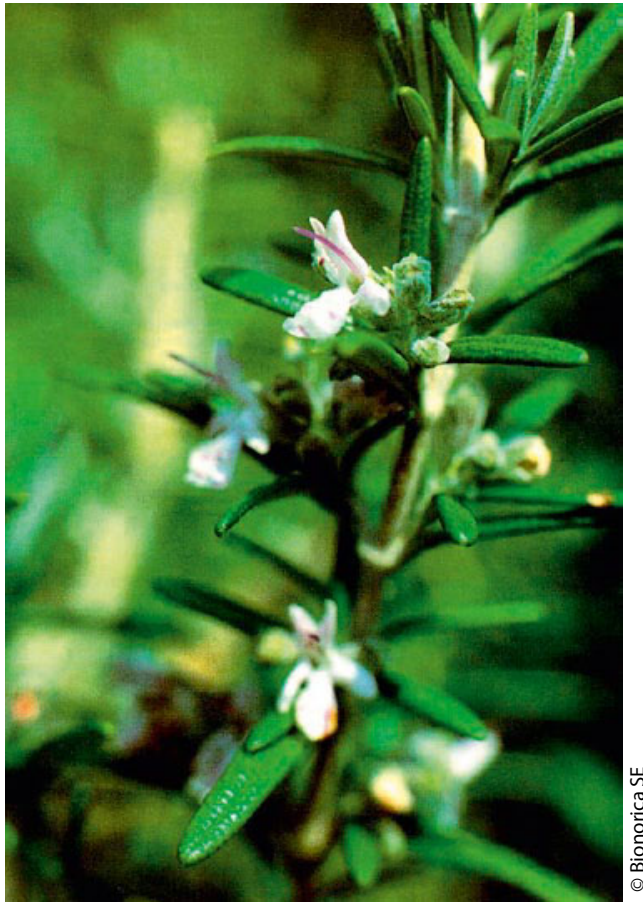

Rosmarin (Rosmarinus officinalis) wirkt antiphlogistisch.
- Canephron ${ }^{\circledast} \mathrm{N}$, die pflanzliche Dreierkombination aus Tausendgüldenkraut, Liebstöckelwurzel und Rosmarinblättern hat ein breites pharmakologisches Wirkspektrum. Das Medikament wird zur Behandlung von leichten Harnwegsinfekten sowie bei rezidivierenden Harnwegsinfekten und Reizblase eingesetzt. Mit dem gut verträglichen Arzneimittel können bereits Jugendliche ab zwölf Jahren behandelt werden.

Neben der antibakteriellen, spasmolytischen und diuretischen Wirkung von Canephron ${ }^{\oplus} \mathrm{N}$ rückte auch die antientzündliche Wirkung des Präparates in den Fokus verschiedener Studien. Zwei wissenschaftliche Untersuchungen belegten in vivo die Wirkung von Rosmarinextrakt und in vitro die der Rosmarinsäure bei entzündlichen Prozessen.

Aus den Ergebnissen einer Untersuchung an menschlichen Zellkulturen schlossen Scheckel et al. 2008, dass Rosmarinsäure die Expression der Cyclooxygenase 2 (COX-2) signifikant verringert. Der Effekt war dosisabhängig.

In einer weiteren Studie von de Farias et al. (2004) wurde in vivo an Ratten der Einfluss eines hydroalkoholischen Rosmarin-Rohextraktes auf den Entzündungsprozess untersucht. Forscher induzierten dabei mit Carrageenin eine Schwellung in der Tierpfote (Rattenpfotenödemtest). Carrageenin fördert die Bildung von Prostaglandin E2 über die Aktivierung der COX-2 und somit den Entzündungsprozess. Vor der lokalen Injektion in die Pfote wurde der Verumgruppe Rosmarinextrakt verabreicht. Die Ergebnisse zeigten, dass Rosmarin dosisabhängig die entzündliche Schwellung reduzierte.

In Canephron ${ }^{\oplus} \mathrm{N}$ wirken nicht nur Rosmarinblätter antiphlogistisch. Auch für Tausendgüldenkraut wurde im Rattenpfotenödemtest eine entsprechende Wirkung gezeigt [Berkan et al., 1989]. Durch die nachgewiesene antientzündliche Wirkung ist Canephron ${ }^{\circledast} \mathrm{N}$ zur Behandlung von Harnwegsinfekten geeignet: Infektbedingtes Brennen und Schmerzen beim Wasserlassen werden effektiv bekämpft. In Verbindung mit der spasmolytischen, diuretischen und antibakteriellen Wirksamkeit hilft Canephron ${ }^{\circledR} \mathrm{N}$ Patienten mit Reizblase oder Symptomen von Harnwegsinfekten somit vierfach und ist dabei gut verträglich.

Nach Informationen von

Bionorica SE, Neumarkt

\section{Zweitlinientherapie beim Prostatakarzinom richtig managen}

- Beim metastasierten hormonresistenten Prostatakarzinom (mHRPC) ist Docetaxel in der Erstlinientherapie der derzeitige Therapiestandard. Nach Docetaxel-Versagen gab es lange Zeit keine zugelassene Therapieoption, erläuterte Dr. Jörg Klier, Köln.„,Andererseits gab es aber Patienten mit einem klaren Therapiewunsch und größtenteils noch einem guten Performance-Status", so Klier. Das neue Taxan Cabazitaxel (Jevtana ${ }^{\circledR}$ ) solle nun die Möglichkeit eröffnen, dort anzusetzen, wo man mit Docetaxel nicht mehr weiterkommt.

In der zulassungsrelevanten TROPIC-Studie (Treatment of Hormone-Refractory Metastatic Prostate Cancer Previously Treated With a Taxotere-Containing Regimen) verringerte sich bei Patienten mit mHRPC und progredientem Verlauf unter Docetaxel das relative Mortalitätsrisiko mit Cabazitaxel (primärer Endpunkt "Overall survival“ OS:
15,1 Monate) um 30\% im Vergleich zu Mitoxantron (OS: 12,7 Monate) [de Bono JS et al. Lancet 2010; 376: 1147-54]. Die häufigsten Nebenwirkungen von Grad 3-4, die mit durchschnittlich $5 \%$ unter Cabazitaxel ( $25 \mathrm{mg} / \mathrm{m}^{2}+$ Prednison, q3w über 10 Zyklen) häufiger auftraten als unter Mitoxantron, waren Diarrhö, Fatigue, Anämie, Neutropenie und febrile Neutropenie.

Mit einem proaktiven Nebenwirkungsmanagement lassen sich laut Dr. Burkhard Otremba, Oldenburg, die Chemotherapiebedingten Nebenwirkungen gut handhaben. Wichtig sei es, so Otremba, den Patienten ausführlich aufzuklären und die möglichen Nebenwirkungen im Vorfeld anzusprechen. Viele Nebenwirkungen ließen sich durch medikamentöse Maßnahmen verhindern, zum Beispiel durch G-CSF-Gabe bei erhöhtem Neutropenierisiko, durch Antibiose bei Infektionen oder durch eine topische antimykotische Prophylaxe bei leichter Stomatitis. Nach Worten von Otremba sind die Nebenwirkungen der Substanz bekannt und beherrschbar, die Patientenauswahl ist aber entscheidend. Wer also sollte Cabazitaxel erhalten? Die Indikationsstellung sollte gemäß der Zulassung, der Allgemeinzustand sollte ausreichend bis gut, die Leber- und Nierenfunktion sollte adäquat sein. Begleiterkrankungen sollten vor allem bei älteren Patienten beachtet werden und es sollte eine ausreichende hämatopoetische Reserve bestehen.

Dr. Claudia Mäck

Satellitensymposium „Behandlung des metastasierten Prostatakarzinoms in der Praxis verlängertes Therapiekontinuum bietet neue Chancen", im Rahmen des 63. DGUKongresses, Hamburg, 15. September 2011 Veranstalter: Sanofi-Aventis, Frankfurt 